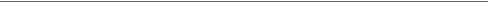

Case Report

\title{
Uterine lipoma: a very rare cause of postmenopausal bleeding
}

\begin{abstract}
The incidence of uterine fatty tumors varies from $0.03-0.2 \%$. The clinical manifestations do not usually differ greatly to those caused by leiomyomas except that they usually affect postmenopausal women. Diagnosis is accomplished after histopathological analysis. A 50 year old post menopausal lady presented with complaints of postmenopausal irregular vaginal bleeding since 1 year. She was P12 L11. On examination, patient was obese, per speculum exam showed hypertrophied cervix with bleeding through os. Bimanual exam was suggestive of a retroverted bulky uterus. Ultrasound Add suggestive of 5.3x6.4x5.8 $\mathrm{cm}$ intramural fibroid, endometrium was not seen. Endometrial biopsy was inconclusive. She underwent total abdominal hysterectomy with bilateral salphingo-opherectomy. Histopathological report was suggestive of uterine lipoma.
\end{abstract}

Keywords: Uterine lipoma, Postmenopausal bleeding, Lipoleiomyoma, Tumor
Volume 9 Issue I - 2018

\author{
Reshika Gangatirkar,' Sandhya Gadre,' \\ Sushruta Shrivastava,' Manal Ali² \\ 'Department of Obstetrics \& Gynecology, Chirayu Medical \\ College \& Bhopal, Barkatullah University, India \\ 2Department of Pathology, Chirayu Medical College \& Bhopal, \\ Barkatullah University, India
}

Correspondence: Dr. Sandhya Gadre, Department of Obstetrics \& Gynecology, Chirayu Medical College \& Bhopal, Barkatullah University, Bhopal, 90, Malviya Nagar, Bhopal, MP, India,Tel 91-9425624449, Email gadre.sandhya@gmail.com

Received: January 15, 2018 | Published: February 14, 2018
Abbreviations: OPD, Out Patient Department; HMGA2, High-Mobility Group AT-hook 2; LPP, Lipoma-Preferred Partner

\section{Introduction}

Benign lipomatous tumors of the uterus are known as lipoma when they only contain fat cells and as lipoleiomyoma when they contain smooth muscle as well as fat cells. ${ }^{1}$ The incidence of uterine fatty tumors varies from $0.03-0.2 \%{ }^{2}$ The diagnosis of pure lipoma should only be made when smooth muscle, if any, is confined to the periphery of the tumour. ${ }^{3}$ The clinical manifestations do not usually differ greatly to those caused by leiomyomas except that they usually affect postmenopausal women. ${ }^{4}$ Most lipomas are located in the body of the uterine corpus. Diagnosis is accomplished after histopathological analysis, although some radiological techniques may indicate their existence prior to surgery. ${ }^{5}$ They have an excellent prognosis.

\section{Case history}

A 50 year old post menopausal lady presented to OPD with complaints of postmenopausal irregular vaginal bleeding since 1 year. She had continuous spotting per vaginum since last 15-20 days. She was P12 L11, all full term vaginal deliveries, attained menopause 4 years back.

On examination, patient was obese, per speculum exam showed hypertrophied cervix with bleeding through os.On bimanual examination uterus was bulky \& retroverted. Ultrasound was suggestive of $5.3 \times 6.4 \times 5.8 \mathrm{~cm}$ intramural fibroid and endometrium was not seen.

Endometrial biopsy of patient was done, which was inconclusive. She underwent total abdominal hysterectomy with bilateral salphingoopherectomy. Postoperative period was uneventful.

On gross appearance, uterus was bulky and uniformly enlarged. $\mathrm{B} / \mathrm{L}$ tubes and ovaries were normal. Cut section showed a $5 \times 5 \mathrm{~cm}$ submucosal yellowish cheesy mass arising from posterior uterine wall (Figures $1 \& 2$ ).

Histopathological report showed thinned out endometrium with few atrophic glands in a compact stroma. Growth in endometrial cavity showed shows an encapsulated lesion composed of lobules of mature adipocytes, separated by thin fibrous septae. No evidence of malignancy.

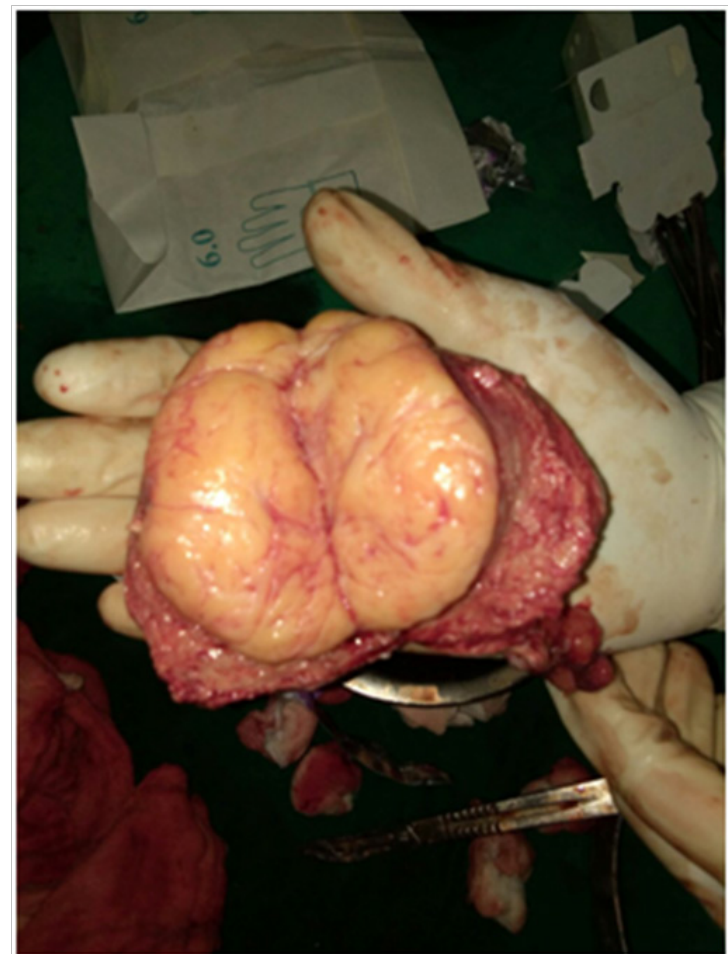

Figure I Gross Specimen: Cut section of uterus with lipoma in situ.

\section{Discussion}

Pure lipomatous tumors of uterus are very rare. They have to be differentiated from more common mixed tumors such as lipofibroma, lipoleiomyoma and fibromyolipoma.

Pure lipoma shows well encapsulated mature adipocytes interspersed by thin fibrous septae. Myometrium is thinned out with few fibers of smooth muscle cells at the periphery of the tumor. It can 
be confused preoperatively with leiomyoma (most common), ovarian teratoma or lipoleiomyoma.

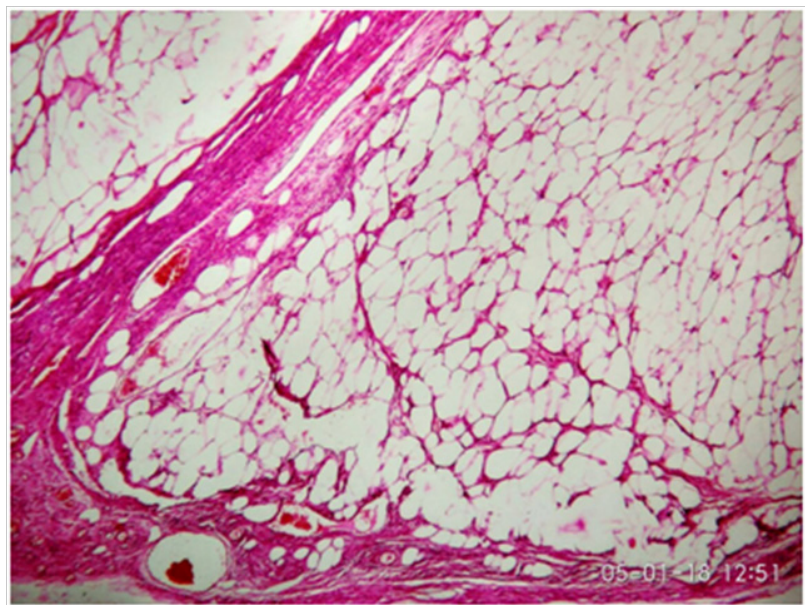

Figure $\mathbf{2}$ Lobules of mature adipocytes, separated by thin fibrous septae.

Imaging techniques offer little help in accurate diagnosis as only few isolated reports diagnosed on MRI are available. The clinical manifestations are similar to leiomyoma, except that pure lipoma is seen more commonly in post menopausal women, with $90 \%$ occurring in patients above 40 years.

In our case, clinical and radiological diagnosis was leiomyoma. This could not explain the occurence of postmenopausal bleeding. Hence histopathological report was eagerly awaited. This turned out to be lipoma, explaining every sign \& symptom. The histogenesis of these lipomatous tumors in the uterine wall continues to be an enigma. As fat tissue is not native to the uterus, various theories of histogenesis have been proposed. These include misplaced embryonic fat cells, metaplasia of the muscle or connective tissue cells into the fat cells, lipocytic differentiation of specific primitive connective tissue cells, proliferation of perivascular fat cells accompanying the blood vessels into the uterus, inclusion of the fat cells into the uterine wall during surgery or fatty infiltration or degeneration of the connective tissue. ${ }^{4}$ The fact that most patients are postmenopausal women leads to the suggestion that fat metabolism changes in this stage may play a role in lipomatous metaplasia. ${ }^{6}$

Pure lipomas can attain huge size before coming into clinical attention and are usually mistaken for carcinomas due to the old age of the patient, rapid progression, and pressure effects. The diagnosis of primary pure lipoma on histopathology should be made only if the smooth muscle cells are confined to the periphery. ${ }^{?}$

In recent years, characteristic chromosomal abnormalities have been found in adipose tumors. Lipomas are frequently characterized by aberrations of the $12 \mathrm{q} 13$ approximately $\mathrm{q} 15$ chromosomal region and often by rearrangements of the HMGA2 gene. These rearrangements include the formation of chimeric genes that fuse the 50 region of HMGA2 with a variety of partners, such as LPP (3q28) or NFIB (9p22). ${ }^{8}$

\section{Acknowledgements}

None.

\section{Conflicts of interest}

None.

\section{References}

1. Resta L, Maiorano E, Piscitelli D, et al. Lipomatous tumors of the uterus: clinico-pathological features of 10 cases with immunocytochemical study of histogenesis. Pathol Res Pract. 1994;190(4):378-383.

2. Garg A, Sudhamani S, Kiri VM, et al. Pure lipoma of uterus: A case report with review of literature. J Sci Soc. 2013;40(2):114-115.

3. Fernandes H, Naik CN, Swethadri GK, et al. Pure lipoma of the uterus: A rare case report. Indian J Pathol Microbiol. 2007;50(4):800-801.

4. Villalonga R, Garcia A. Lipoma of the Uterine Corpus: Exceptional Eventuality Combined with an Ovarian Thecoma. Case Rep Med. 2009;2009:340603.

5. Houser LM, Carasco CH, Seehan CR. Lipomatous tumour of the uterus: radiographic and ultrasonic appearance. Br J Radiol. 1979;52(624):992993

6. Lin $\mathrm{KC}$, Sheu BC, Huang SC. Lipoleiomyoma of the uterus. Int $J$ Gynaecol Obstet. 1999;67(1):47-49.

7. Vamseedhar A, Shivalingappa DB, Suresh DR, et al. Primary pure uterine lipoma: A rare case report with review of literature. Indian $J$ Cancer. 2011;48(3):385-387.

8. Pellegrino A, Damiani GR, Pirovano C, et al. A rare case of pure uterine giant lipoma. Taiwan J Obstet Gynecol. 2016;55(1):145-146. 